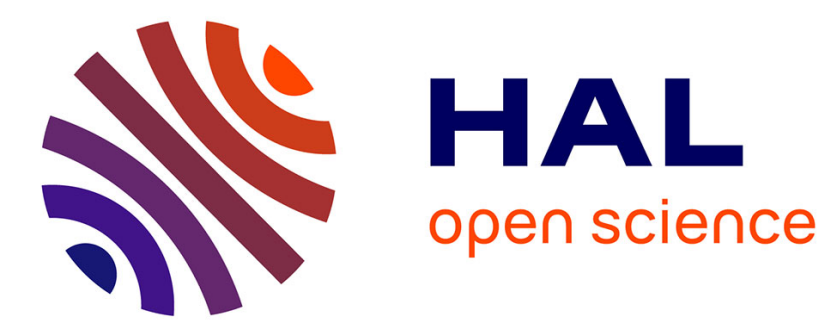

\title{
OBSERVATIONS DES RAIES DANS LE DOMAINE RADIO
}

\author{
F. Combes, P. Encrenaz
}

\section{To cite this version:}

F. Combes, P. Encrenaz. OBSERVATIONS DES RAIES DANS LE DOMAINE RADIO. Journal de Physique Colloques, 1978, 39 (C1), pp.C1-131-C1-135. 10.1051/jphyscol:1978122 . jpa-00217318

\section{HAL Id: jpa-00217318 https://hal.science/jpa-00217318}

Submitted on 1 Jan 1978

HAL is a multi-disciplinary open access archive for the deposit and dissemination of scientific research documents, whether they are published or not. The documents may come from teaching and research institutions in France or abroad, or from public or private research centers.
L'archive ouverte pluridisciplinaire HAL, est destinée au dépôt et à la diffusion de documents scientifiques de niveau recherche, publiés ou non, émanant des établissements d'enseignement et de recherche français ou étrangers, des laboratoires publics ou privés. 


\title{
OBSERVATIONS DES RAIES DANS LE DOMAINE RADIO
}

\author{
F. COMBES et P. J. ENCRENAZ
}

Laboratoire de Physique, Ecole Normale Supérieure

et

Département de Radioastronomie, Observatoire de Meudon, 92190 Meudon, France

\begin{abstract}
Résumé. - Le milieu interstellaire se révèle très riche en raies dans le domaine centimétrique et millimétrique, depuis la transition hyperfine de l'atome d'hydrogène qui a permis d'explorer la galaxie, jusqu'aux très nombreuses raies de rotation des molécules. L'observation de CO, la molécule la plus abondante après $\mathrm{H}_{2}$, et de son isotope ${ }^{13} \mathrm{CO}$ permet d'atteindre la densité et la température des nuages denses. Les émissions maser $\left(\mathrm{H}_{2} \mathrm{O}, \mathrm{OH}, \mathrm{SiO}\right)$ ou antimaser $\left(\mathrm{H}_{2} \mathrm{CO}\right)$ révèlent les conditions spéciales d'excitation de certains milieux. L'identification de molécules organiques complexes (jusqu'à 9 atomes) pose le problème de leur formation et de leur abondance.
\end{abstract}

\begin{abstract}
The interstellar medium is very rich in centimetric and millimetric lines, from the hyperfine transition of the atomic hydrogen, which yields the galactic structure, to the numerous molecular rotation lines. Emission from $\mathrm{CO}$, the most abundant molecule after $\mathrm{H}_{2}$, and from ${ }^{13} \mathrm{CO}$, can yield the density and temperature in dense molecular clouds. From the maser emission $\left(\mathrm{H}_{2} \mathrm{O}\right.$, $\mathrm{OH}, \mathrm{SiO})$ or antimaser emission $\left(\mathrm{H}_{2} \mathrm{CO}\right)$, special excitation conditions are deduced in some mediums. The identification of complex organic molecules ( 9 atoms) rises the question of their formation and abundance.
\end{abstract}

La première détection d'une raie dans le domaine radio fut celle du constituant le plus abondant dans l'univers, l'hydrogène (Ewen et Purcell, 1951). Il s'agit de la raie à $21 \mathrm{~cm}(1420 \mathrm{MHz})$ correspondant à la transition $F=1-0$ entre deux sous-niveaux hyperfins de l'état fondamental de l'hyđ̛rogène atomique. Elle a permis d'explorer la structure spirale de la Galaxie. L'hydrogène neutre représente entre 5 et $10 \%$ de la masse totale d'une galaxie spirale. En première approximation, le gaz a une température de $100 \mathrm{~K}$ et une densité de 1 à $10 \mathrm{at} / \mathrm{cm}^{3}$ dans les bras spiraux et $0,1 \mathrm{at} / \mathrm{cm}^{3}$ entre les bras. Une étude plus raffinée conduit à un modèle à deux composants pour le milieu interstellaire : l'un consiste en nuages froids et denses, $T \leqslant 100 \mathrm{~K}$ et $n \sim 10-100 \mathrm{at} / \mathrm{cm}^{3}$, l'autre en nuages diffus et chauds, $T \sim 10^{4} \mathrm{~K}$ et $n \sim 0,1 \mathrm{at} / \mathrm{cm}^{3}$.

La deuxième raie radio, découverte en 1963 , fut celle $\mathrm{du}$ radical $\mathrm{OH}$ à $18 \mathrm{~cm}$. La structure hyperfine $\mathrm{du}$ niveau ${ }^{2} \Pi_{3 / 2}$ donne lieu à deux raies principales à $1665 \mathrm{MHz}(\mathrm{F}=1-1)$ et $1667 \mathrm{MHz}(\mathrm{F}=2-2)$ et deux raies satellites à $1720 \mathrm{MHz}(\mathrm{F}=2-1)$ et $1612 \mathrm{MHz}(\mathrm{F}=1-2)$. Le radical $\mathrm{OH}$ est très étendu dans la Galaxie où on le voit en absorption devant des sources continu (régions HII galactiques ou sources extragalactiques). OH est aussi observé en émission : très souvent l'équilibre thermodynamique est loin d'être atteint, les populations des quatre niveaux sont fortement perturbées et certaines des quatre raies se présentent en absorption, les autres en émission, au même point. Les raies du radical $\mathrm{OH}$ sont souvent détectées en émission maser; elles sont étroites, très intenses, donc requièrent une grande source d'énergie, elles sont polarisées et variables rapidement, sur une échelle de temps de l'ordre de la semaine, donc sont limitées spatialement. En général ce sont des milieux confinés à l'atmosphère de jeunes étoiles froides, émettant en infra-rouge (étoiles de type Mira) [1], ou d'étoiles en formation. Lorsque la source se trouve dans l'enveloppe en expansion d'une étoile, le profil a deux composantes bien séparées en vitesse. Des modèles de pompage infra-rouge et collisionnel ont été étudiés [2], [3], [4]. Ces modèles nécessitent des densités supérieures à $10^{8}$ at $/ \mathrm{cm}^{3}$ dans le milieu.

L'effet Zeeman serait intéressant à observer avec l'émission $\mathrm{OH}$ thermique des nuages de poussière [5]. Seules des limites sur le champ magnétique ont pu être observées jusqu'à présent : $30 \mu \mathrm{G}$ dans CAS A et $130 \mu \mathrm{G}$ dans le nuage $4 \mathrm{C}$.

Les découvertes de raies moléculaires dans le domaine radio se sont précipitées à partir de 1968 , notamment $\mathrm{H}_{2} \mathrm{O}, \mathrm{NH}_{3}$ et $\mathrm{H}_{2} \mathrm{CO}$ en 1969. On n'espérait pas la présence de molécules si élaborées dans le milieu interstellaire, peu dense où la probabilité de collisions est très faible, donc les réactions chimiques peu favorisées. La solution se trouve peut-être dans la formation à la surface des grains de poussière, pour la molécule $\mathrm{H}_{2}$ notamment, ou dans les réactions ionsmolécules et réactions en phase gazeuse entre neutres. Notons que la formation des molécules dans le milieu interstellaire est surtout efficace pour les molécules organiques. 


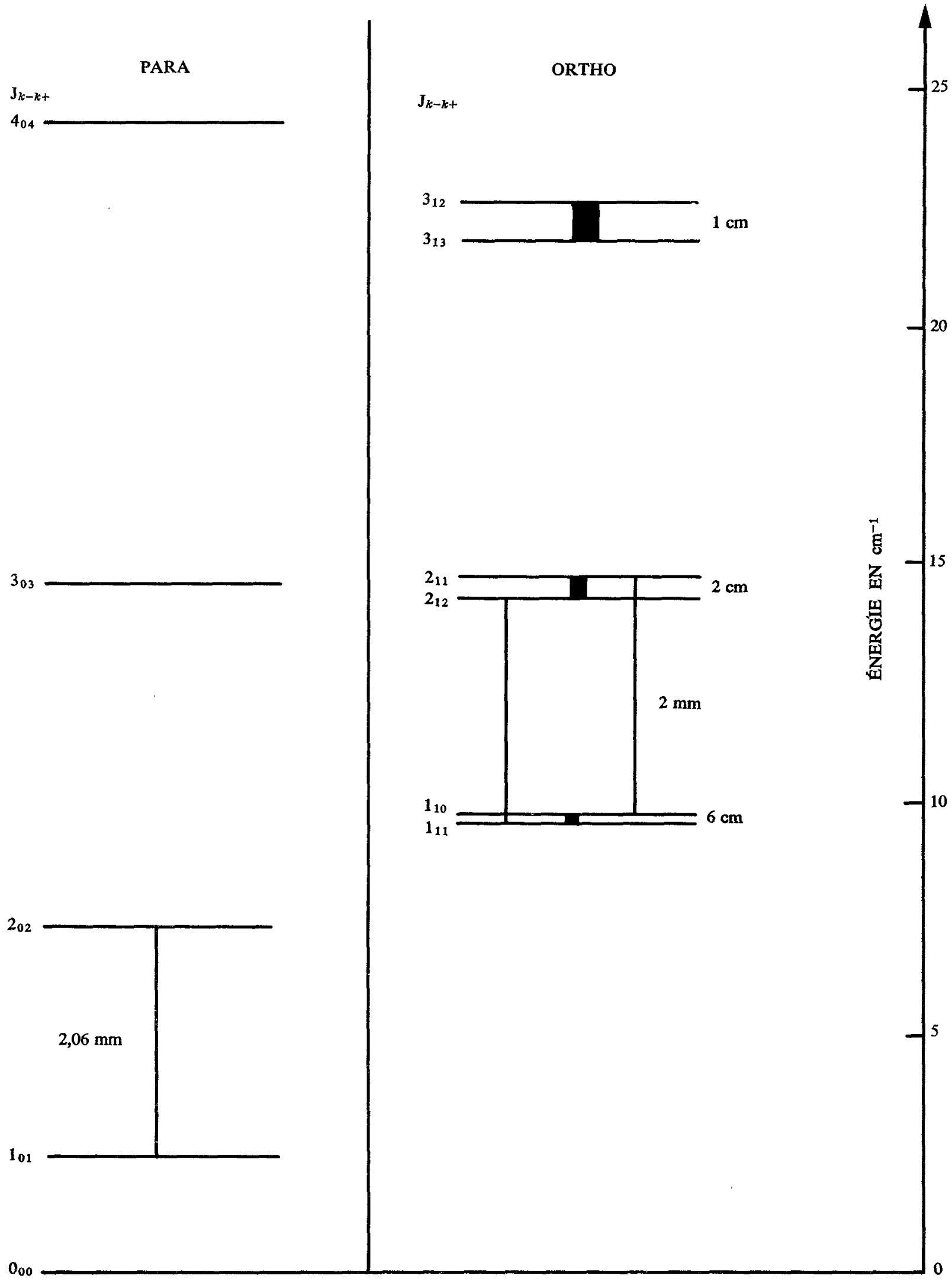

FIG. 1. - Schéma des niveaux du formaldéhyde $\mathbf{H}_{2} \mathrm{CO}$. 
a) $\mathrm{H}_{2} \mathrm{O}$ : La raie tombe dans le domaine radio grâce à une coïncidence fortuite entre les niveaux $6_{16}$ et $5_{23}$ situés à $447 \mathrm{~cm}^{-1}$ du niveau fondamental, qui ne sont séparés que par $22 \mathrm{GHz}(1,35 \mathrm{~cm})$. La raie correspond donc à une température d'excitation très élevée et elle n'est détectée qu'en émission maser. Ces masers sont parfois observés au voisinage des masers $\mathrm{OH}$, et comme eux sont rapidement variables, sūr une échelle de temps descendant à quelques jours ; l'interférométrie à très longue base confirme la petite taille des régions émettrices $\left(5 \times 10^{-3 \prime}\right.$ dans Orion). Les températures de brillance peuvent atteindre $10^{13} \mathrm{~K}$.

b) $\mathrm{NH}_{3}$ : La fréquence d'inversion de l'ammoniac est très bien connue en laboratoire (maser à ammoniac) : $23,7 \mathrm{GHz}$. Townes découvrit les raies correspondantes en direction du centre galactique en 1969 . $\mathrm{NH}_{3}$ est aussi observé en absorption.

c) $\mathrm{H}_{2} \mathrm{CO}$ : Snyder, Buhl, Zuckerman, Palmer découvrent $\mathrm{H}_{2} \mathrm{CO}$ dans toute la Galaxie en 1969 [6]. Les premiers niveaux de rotation $\mathrm{du}$ formaldéhyde ortho présentent les transitions à $6 \mathrm{~cm}$ $\left(\mathrm{J}_{k-k+}=1_{10}-1_{11}\right)$ et $2 \mathrm{~mm}\left(\mathrm{~J}_{k-k+}=2_{12}-1_{11}\right)$ (voir Fig. 1). La raie $2 \mathrm{~mm}$ est observée en émission, mais la raie à $6 \mathrm{~cm}$ l'est en absorption, même en l'absence de toute source locale de continuum : seul le rayonnement du fond cosmologique à $2,7 \mathrm{~K}$ peut être absorbé, la température d'excitation est donc inférieure à $2,7 \mathrm{~K}$. Il s'agit d'un réfrigérateur, où les atomes sont pompés du niveau 2 au niveau 1 .

L'étude du transfert de rayonnement pour cette molécule a été effectuée par $\mathrm{R}$. Lucas [7]. Les sections efficaces de collision $\mathrm{H}_{2}-\mathrm{H}_{2} \mathrm{CO}$, calculées purement quantiquement [8], permettent d'expliquer l'antiinversion: une des sections efficaces dépeuplant le niveau 2 au profit de niveaux supérieurs, qui par désexcitation radiative peuplent le niveau 1 , est bien supérieure à la section efficace correspondante pour le niveau $1\left(\sigma_{27} / \sigma_{16} \sim 10\right)$. La densité $n$ doit obéir à deux conditions :

- être assez forte pour que les collisions luttent efficacement contre l'absorption du fond continu des niveaux 1 à $2: n>500 \mathrm{~cm}^{-3}$,

- ne pas être trop forte pour éviter la thermalisation des raies : $n<5 \times 10^{5} \mathrm{~cm}^{-3}$.

Le modèle utilisé par R. Lucas est un nuage uniforme en température et densité, sans gradient de vitesse mais agité de turbulence. Les huit premiers niveaux du spectre sont pris en compte. Les paramètres physiques sont fournis par l'observation des raies à $6 \mathrm{~cm}$, avec le télescope d'Effelsberg (lobe de 2,6'), et à $2 \mathrm{~mm}$, avec le télescope de l'observatoire millimétrique de Fort Davis, Texas (lobe de $2^{\prime}$ ) ; un exemple des spectres obtenus est présenté en figure 2. Pour chaque raie observée, le modèle donne une relation entre la densité de formaldéhyde sur la ligne de visée et la densité volu-

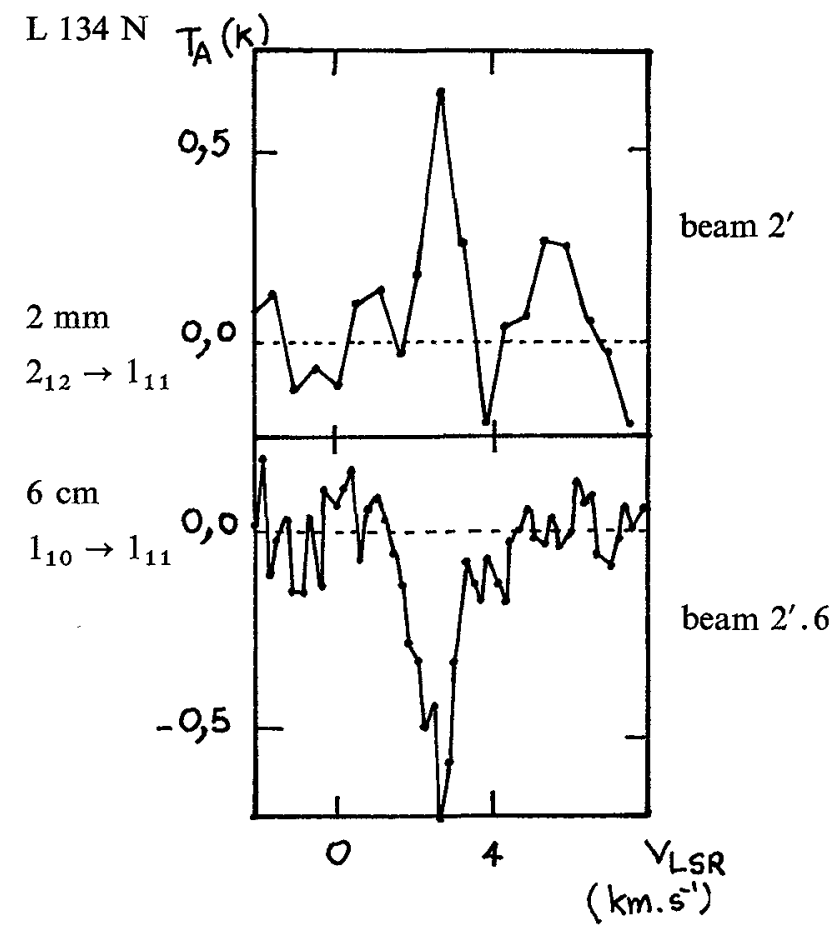

Fig. 2. - Spectres observés à la position centrale du nuage L134N [7]. Les positions et intensités relatives des composantes hyperfines de la transition à $6 \mathrm{~cm}$ sont indiquées.

mique d'hydrogène moléculaire. Les deux courbes représentant ces deux relations doivent avoir un point d'intersection, donnant les valeurs de ces densités $\left(N_{\mathrm{H}_{2} \mathrm{CO}}\right.$ et $\left.n_{\mathrm{H}_{2}}\right)$ pour le nuage considéré (voir Fig. 3).

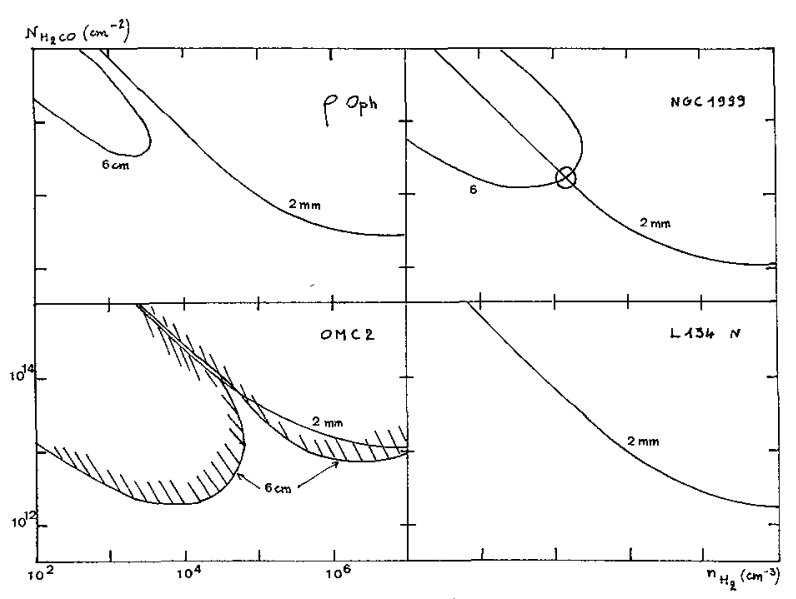

FIG. 3. - Diagrammes $\left(n_{\mathrm{H}_{2}}, N_{\mathrm{H}_{2} \mathrm{CO}}\right)$ pour quatre des positions observées [7].

Une seule incompatibilité est observée compte tenu des incertitudes sur les sections de choc, c'est le cas du nuage $\mathrm{L} 134 \mathrm{~N}$ : la raie à $6 \mathrm{~cm}$ observée est très intense et le modèle n'en peut rendre compte. Ce désaccord ne dépend pas du modèle de vitesse choisi pour le nuage, et semble mettre en difficulté le modèle de collisions de Garrison et al. [8]. 
d) CO: La molécule COest largement distribuée dans la Galaxie, c'est la plus abondante après $\mathrm{H}_{2}$; elle est un outil précieux pour tracer les régions denses et la structure spirale. Sa distribution radiale consiste en une région annulaire de 4 à $8 \mathrm{kpc}$ et son épaisseur à mi-hauteur est de $59 \mathrm{pc}$ [9]. La transition $\mathrm{J}=1-{ }^{\prime} 0$ $(115 \mathrm{GHz}) \mathrm{de}{ }^{12} \mathrm{CO}$ est pratiquement toujours saturée dans les nuages denses, aussi indique-t-elle la température d'excitation. La donnée conjuguée de la raie correspondante de ${ }^{13} \mathrm{CO}$, en général optiquement mince, permet de déterminer l'abondance de CO sur la ligne de visée. Un exemple intéressant de nuage dense est le nuage situé dans $\rho$ Ophiucus : c'est un nuage très étendu, de 1 à $2^{\circ}$, comportant plusieurs maxima dans l'émission de ${ }^{12} \mathrm{CO}$ [10]. Il a été détecté de nombreuses sources infra-rouge par Vrba et al. [11] et plusieurs sources continu à $11 \mathrm{~cm}$ ont été découvertes avec l'interféromètre de Westerbork par P. J. Encrenaz, E. Falgaronne et R. Lucas [10]. Le rapport d'abondance $\mathrm{CO} / \mathrm{H}_{2}$ a pu être déterminé dans ce nuage par la méthode suivante :

- l'absorption visuelle $A_{\mathrm{v}}$ en tout point du nuage est déterminée par des comptages d'étoiles ; on peut en déduire, à l'aide des observations ${ }^{12} \mathrm{CO}$ et ${ }^{13} \mathrm{CO}$, le rapport $N_{\mathrm{co}} / A_{\mathrm{v}}\left(\mathrm{cm}^{-2} / \mathrm{mag}\right.$.) en fonction de $A_{\mathrm{v}}$ (voir Fig. 4),

- d'après le rapport $N_{\mathrm{H}_{2}} / A_{\mathrm{v}}\left(\mathrm{cm}^{-2} / \mathrm{mag}\right.$.) en fonction de $A_{v}$, déterminé par l'observation en ultraviolet (Copernicus), on peut en déduire le rapport d'abondance $\mathrm{CO} / \mathrm{H}_{2}$. Ce rapport, qui est dans $\rho$ Ophiucus de $\sim 10^{-4}$, semble garder la même valeur dans d'autres nuages denses, à peu de variations près.

Des raies de recombinaison du carbone du domaine radio (correspondant à des nombres quantiques $n$ élevés) ont été observées dans $\rho$ Ophiucus [12], de même que des raies de recombinaison du soufre. D'après les caractéristiques des régions $\mathrm{C}$ II et $\mathrm{S}$ II, il est possible d'évaluer en résolvant le transfert, la puissance et le type spectral de l'étoile excitatrice [13].

De nombreuses autres molécules ont des transitions de leur spectre de rotation dans le domaine millimé-

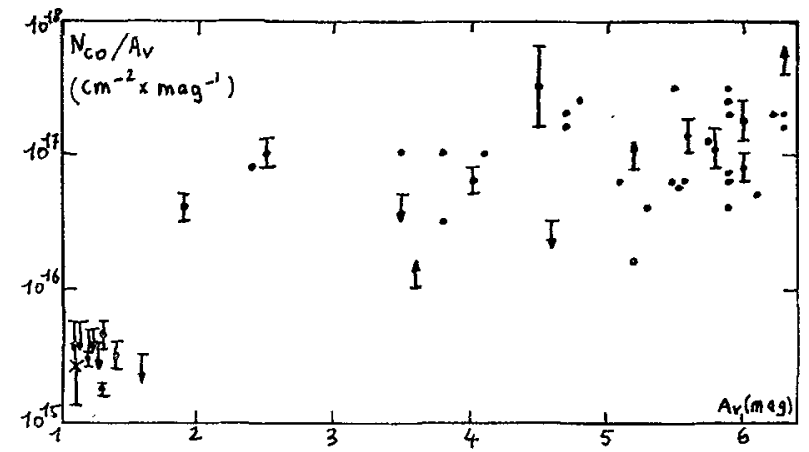

FIG. 4. $-N_{\mathrm{CO}} / A_{\mathrm{V}}$ en fonction de $A_{\mathrm{v}}$ pour les points où $A_{\mathrm{V}} \leqslant$ 6,3 mag. [10]. Les barres d'erreur sont dues à l'incertitude sur $T_{k}$ pour les points où une seule composante isotopique fut observée.

trique : l'énergie d'un niveau rotationnel étant proportionnelle à $1 / I_{0}$, i.e. à l'inverse du moment d'inertie. Le domaine millimétrique est très riche, 200 transitions ont été observées jusqu'à présent, correspondant à plus de 40 molécules. Comment sont excitées ces molécules ? Les collisions électroniques sont très efficaces, mais les électrons ne sont pas assez abondants dans les nuages denses; leur densité $n_{\mathrm{e}}$ est évaluée à $10^{-2} \mathrm{~cm}^{-3}$ (soit d'après un taux d'ionisation $\xi=10^{-17} \mathrm{~s}^{-1}$ généralement adopté pour le milieu interstellaire, soit d'après les mesures de dispersion des pulsars). Les collisions avec l'hydrogène moléculaire sont donc prédominantes. L'émission dans une raie moléculaire dépendra d'une façon assez complexe de la densité $n_{\mathrm{H}_{2}}$ et du moment dipolaire de la molécule : il y a compétition entre l'excitation et la désexcitation collisionnelles et l'émission spontanée [14]. On peut en déduire qu'en général les molécules à moment dipolaire élevé requièrent une densité plus forte d'hydrogène moléculaire pour être excitées (par exemple, $n_{\mathrm{H}_{2}}$ de l'ordre de $10^{3} \mathrm{~cm}^{-3}$ pour $\mathrm{CO}$ et $10^{5} \mathrm{~cm}^{-3}$ pour $\mathrm{HCN}$ ).

La liste des molécules observées jusqu'en Mai 1977 est présentée en table $\mathrm{I}$. $\mathrm{OH}, \mathrm{H}_{2} \mathrm{O}$ et $\mathrm{SiO}$ sont observées en émission maser.

TABLE I

Molécules observées (Mai 1977)

\begin{tabular}{|c|c|c|c|c|c|c|c|}
\hline 2 & 3 & 4 & 5 & 6 & 7 & 8 & 9 \\
\hline $\mathrm{H}_{2}$ & $\mathrm{H}_{2} \mathrm{O}$ & $\mathrm{H}_{2} \mathrm{CO}$ & $\mathrm{HCOOH}$ & $\mathrm{HCH}_{2} \mathrm{OH}$ & $\mathrm{CH}_{3} \mathrm{C}_{2} \mathrm{H}$ & $\mathrm{CH}_{3} \mathrm{COOH}$ & $\left(\mathrm{CH}_{3}\right)_{2} \mathrm{O}$ \\
\hline $\mathrm{CH}$ & $\mathrm{HCO}$ & $\mathrm{NH}_{3}$ & $\mathrm{HC}_{3} \mathrm{~N}$ & $\mathrm{NH}_{2} \mathrm{CHO}$ & $\mathrm{CH}_{3} \mathrm{CHO}$ & & $\mathrm{CH}_{3} \mathrm{CH}_{2} \mathrm{OH}$ \\
\hline $\mathrm{CH}^{+}$ & $\mathrm{HCO}^{+}$ & $\mathrm{HNCO}$ & $\mathrm{CH}_{2} \mathrm{NH}$ & $\mathrm{CH}_{3} \mathrm{CN}$ & $\mathrm{HC}_{5} \mathrm{~N}$ & & $\mathrm{CH}_{3} \mathrm{CH}_{2} \mathrm{CN}$ \\
\hline $\mathrm{CN}$ & $\mathrm{CCH}$ & $\mathrm{H}_{2} \mathrm{CS}$ & $\mathrm{NH}_{2} \mathrm{CN}$ & & $\mathrm{CH}_{3} \mathrm{NH}_{2}$ & & $\mathrm{HC}_{7} \mathrm{~N}$ \\
\hline $\mathrm{CO}$ & HCN & $\mathrm{C}_{3} \mathrm{~N}$ & $\mathrm{H}_{2} \mathrm{CCO}$ & & $\mathrm{CH}_{2} \mathrm{CHCN}$ & & \\
\hline CS & HNC & & & & & & \\
\hline $\mathrm{OH}$ & $\mathrm{N}_{2} \mathrm{H}^{+}$ & & & & & & \\
\hline so & $\mathrm{H}_{2} \mathrm{~S}$ & & & & & & \\
\hline NS & OCS & & & & & & \\
\hline $\mathrm{SiO}$ & $\mathrm{SO}_{2}$ & & & & & & \\
\hline $\mathrm{Si}$ & & & & & & & \\
\hline
\end{tabular}


Isotopes : De nombreuses molécules deutérées ont été détectées: $\mathrm{HD}, \mathrm{HDO}, \mathrm{DCN}, \mathrm{DCO}^{+}, \mathrm{DNC}$, $\mathrm{N}_{2} \mathrm{D}^{+}, \mathrm{NH}_{2} \mathrm{D}$. L'abondance du deutérium est très importante en cosmologie : la nucléosynthèse primordiale produit surtout de l'hélium et du deutérium, dont l'abondance finale dépend des paramètres du modèle cosmologique. Il est donc nécessaire de savoir dans quelles proportions le deutérium est ensuite détruit par astration (i. e. pendant le cycle de la matière qui participe à la formation d'une étoile, puis est rejetée dans le milieu interstellaire), ou enrichi par nucléosynthèse dans les étoiles (par spallation au cours des ondes de choc des explosions de supernovae).

Un des tests pouvant répondre à cette question est la distribution spatiale du deutérium dans la Galaxie : s'il est cosmologique, il se trouve dans les régions peu riches en métaux, à faible astration, sinon dans les régions de formation intense d'étoiles, le centre galactique essentiellement. Wannier et al. en 1976 [15] ont déterminé une abondance de $\mathrm{DCN} / \mathrm{HCN}$ plus faible au centre galactique que dans les régions extérieures, où ce rapport n'a que de faibles variations de source à source. Ces mesures tendent à prouver que le deutérium est cosmologique.

Le rapport $\mathrm{DCN} / \mathrm{HCN}$ mesuré est de $3 \times 10^{-3}$, cent fois supérieur au rapport $\mathrm{D} / \mathrm{H}$ mesuré dans le voisinage du soleil, $3 \times 10^{-5}$ : il s'agit essentiellement de fractionation chimique, l'énergie de liaison de DCN étant supérieure à celle de $\mathrm{HCN}$; la photodissociation de $\mathrm{D}_{2}$ pourrait aussi être plus efficace que celle de $\mathrm{H}_{2}$, $\mathrm{D}$ atomique étant alors plus abondant que $\mathrm{H}$ pour participer aux réactions chimiques. L'abondance du deutérium dans les autres molécules deutérées où elle a pu être mesurée est de $5 \times 10^{-3}$ pour $\mathrm{DCO}^{+}$et $10^{-2}$ pour DNC.

Le rapport ${ }^{12} \mathrm{C} /{ }^{13} \mathrm{C}$, qui est de 89 dans le système solaire, n'est déterminé qu'avec une grande incertitude dans les nuages moléculaires, entre 30 et 90 . La valeur 40 semble être plus souvent observée [16]. Les molécules dans lesquelles ce rapport a été déterminé sont $\mathrm{CO}, \mathrm{HCN}, \mathrm{CS}, \mathrm{H}_{2} \mathrm{CO}$.

L'oxygène a deux isotopes stables ${ }^{17} \mathrm{O}$ et ${ }^{18} \mathrm{O}$, dont les abondances solaires sont respectivement $1 / 2700$ et $1 / 490$ par rapport à l'oxygène ${ }^{16} \mathrm{O}$. Wannier et al. [17] ont mesuré dans les nuages moléculaires un rapport de ${ }^{17} \mathrm{O} /{ }^{18} \mathrm{O}$ supérieur à la valeur terrestre et suggèrent une surabondance de ${ }^{17} \mathrm{O}$ (plutôt qu'une déficience de $\left.{ }^{18} \mathrm{O}\right)$ par des arguments de nucléosynthèse.

L'azote et le soufre ont été observés avec une abondance terrestre par rapport à leurs isotopes.

Les molécules ne cessent de poser des problèmes physiques et chimiques par leur formation, leur excitation, leurs abondances. Elles sont un traceur des régions denses, et les masers $\left(\mathrm{H}_{2} \mathrm{O}, \mathrm{OH}, \mathrm{SiO}\right)$ ont permis de faire le lien entre les molécules et les étoiles jeunes ou en formation.

\section{Bibliographie}

[1] BAUDRY, A., Astron. Astrophys. 32 (1974) 191.

[2] Elitzur, M., Goldreich, P., Scoville, N., Astrophys. $J$. 205 (1976) 384.

[3] Rieu Nguyen-Q, Winnberg, A., Guibert, J., Lepine, J. R. D., Johansson, X. E. B., Goss, W. M., Astron. Astrophys. 46 (1976) 413.

[4] Elitzur, M., Astrophys. J. 203 (1976) 124.

[5] Turner, B. E., Verschudr, G. L., Astrophys, J. 162 (1970) 341.

[6] Palmer, P., Zuckerman, B., Buhl, D., Snyder, L. E., Astrophys. J. 156 (1969) L 147.

[7]. LUCAS, R., Thèse non publiée (1976).

[8] Garrison, B. J., Lester, W. A. Jr, Miller, W. H., Green, S., Astrophys. J. 200 (1975) L 175.

[9] Cohen, R. S., Thaddeus, P. (1977) preprint.
[10] Encrenaz, P. J., Falgaronne, E., Lucas, R., Astron. Astrophys. 44 (1975) 73.

[11] VRBa, F. J., Strom, R. M., Strom, S. E., Grasdalen, G. L., Astrophys. J. 197 (1975) 77.

[12] Cesarsky, D. A., Encrenaz, P. J., Falgaronne, E. G., LAZAREFF, B., LAUQú, R., LuCAS, R., WelIACheW, L.., Astron. Astrophys. 48 (1976) 167.

[13] Falgaronne, E. G. en préparation.

[14] Penzias, A. A. (1974), Cours des Houches.

[15] Wannier, P. G., Linke, R. A., Wilson, R. W., PenZIAS, A. A., preprint.

[16] Wannier, P. G., Penzias, A. A., Linke, R. A., Wilson, R. W., Astrophys. J. 204 (1976) 26.

[17] Wannier, P. G., LuCAS, R., Linke, R. A., Encrenaz, P. J., Penzias, A. A., Wilson, R. W., Astrophys. J. 205 (1976) L 169. 